\title{
New developments in the treatment of obstructive sleep apnoea
}

\author{
Anita K Simonds \\ Sleep \& Ventilation Unit, Royal Brompton \& Harefield NHS Trust, \\ London SW3 6NP, UK
}

Introductory articles

\section{Comparison of therapeutic and subtherapeutic nasal continuous positive airway pressure for obstructive sleep apnoea: a randomised prospective parallel trial}

\author{
C Jenkinson, RJO Davies, R Mullins, JR Stradling
}

Background. Nasal continuous positive airway pressure (NCPAP) is widely used as treatment for $\stackrel{\circ}{\rightarrow}$ obstructive sleep apnoea. However, to date there are no randomised controlled trials of this therapy against a well matched control. We undertook a randomised prospective parallel trial of therapeutic $\frac{6}{5}$ NCPAP for obstructive sleep apnoea compared with a control group on subtherapeutic NCPAP. Methods. Men with obstructive sleep apnoea, defined as an Epworth sleepiness score of 10 or more and ten or 8 more dips per $h$ of more than $4 \% \mathrm{SaO}_{2}$ caused by obstructive sleep apnoea on overnight sleep study, were randomly assigned therapeutic or subtherapeutic NCPAP (about $1 \mathrm{~cm} \mathrm{H}_{2} \mathrm{O}$ ) for 1 month. Primary outcomes were subjective sleepiness (Epworth sleepiness score), objective sleepiness (maintenance of wakefulness test), and SF-36 questionnaire measurements of self-reported functioning and well-being. Findings. 107 men entered the study: 53 received subtherapeutic NCPAP and 54 therapeutic NCPAP. Use $\triangle$ of NCPAP by the two treatment groups was similar: $5.4 \mathrm{~h}$ (therapeutic) and $4.6 \mathrm{~h}$ (subtherapeutic) per night. Subtherapeutic CPAP did not alter the overnight number of $\mathrm{SaO}_{2}$ dips per $h$ compared with baseline, and thus acted as a control. Therapeutic NCPAP was superior to subtherapeutic NCPAP in all primary outcome measures. The Epworth score decreased from a median of 15.5 to 7.0 on therapeutic NCPAP and from 15.0 to 13.0 on subtherapeutic nCPAP (between treatments, $p<0.0001$ ). Mean $\frac{0}{N}$ maintenance of wakefulness time increased from 22.5 to $32.9 \mathrm{~min}$ on therapeutic NCPAP and, not $\times$ significantly, from 20.0 to $23.5 \mathrm{~min}$ on subtherapeutic NCPAP (between treatments, $p<0.005$ ). Effect $\underline{3}$. sizes for SF-36 measures of energy and vitality were 1.68 (therapeutic) and 0.97 (subtherapeutic) NCPAP 8 (between treatments, $p<0.0001$ ). For mental summary score the corresponding values were 1.02 and $₹$ 0.4 (between treatments, $p=0.002$ ). Interpretation. Therapeutic NCPAP reduces excessive daytime 9 sleepiness and improves self-reported health status compared with a subtherapeutic control. Compared $\frac{D}{0}$ with controls, the effects of therapeutic NCPAP are large and confirm previous uncontrolled clinical observations and the results of controlled trials that used an oral placebo. (Lancet 1999;353:2100-5)

\section{Mandibular advancement oral appliance therapy for obstructive sleep apnoea: effect on awake calibre of the velopharynx}

\section{CF Ryan, LL Love, D Peat, JA Fleetham, AA Lowe}

Background. The mechanisms of action of oral appliance therapy in obstructive sleep apnoea are poorly understood. Videoendoscopy of the upper airway was used during wakefulness to examine whether $\stackrel{\otimes}{2}$ the changes in pharyngeal dimensions produced by a mandibular advancement oral appliance are $\sigma$ related to the improvement in the severity of obstructive sleep apnoea. Methods. Fifteen patients with 8 mild to moderate obstructive sleep apnoea (median (range) apnoea index (Al) 4 (0-38)/h, apnoeahypopnoea index (AHI) 28 (9-45)/h) underwent overnight polysomnography and imaging of the upper $\stackrel{0}{\overrightarrow{2}}$ airway before and after insertion of the oral appliance. Images were obtained in the hypopharynx, oropharynx, and velopharynx at end tidal expiration during quiet nasal breathing in the supine position. The cross sectional area and diameters of the upper airway were measured using image processing software with an intraluminal catheter as a linear calibration. Results. Al decreased to a median (range) 
value of $0(0-6) / h(p<0.01)$ and $A H I$ to $8(1-28) / h(p<0.001)$ following insertion of the oral appliance. The median (95\% confidence interval) cross sectional area of the upper airway increased by $18 \%$ (3 to 35) $(p<0.02)$ in the hypopharynx and by $25 \%$ (11 to 69) $(p<0.005)$ in the velopharynx, but not significantly $\vec{\sigma}$ in the oropharynx. Although in general the shape of the pharynx did not change following insertion $\underset{x}{x}$ of the oral appliance, the lateral diameter of the velopharynx increased to a greater extent than the $\vec{\Rightarrow}$ anteroposterior diameter. Following insertion of the oral appliance the reduction in AHI was related to the increase in cross sectional area of the velopharynx $(p=0.01)$. Conclusions. A mandibular $\frac{0}{\sigma}$ advancement oral appliance increases the cross sectional area of the upper airway during wakefulness, $\frac{\bar{v}}{\mathrm{n}}$ particularly in the velopharynx. Assuming this effect on upper airway calibre is not eliminated by sleep, $\stackrel{\mathbb{\Omega}}{2}$ mandibular advancement oral appliances may reduce the severity of obstructive sleep apnoea by maintaining patency of the velopharynx, particularly in its lateral dimension. (Thorax 1999;54:972-7) $\overrightarrow{0}$

\section{Positional treatment vs continuous positive airway pressure in patients with positional obstructive sleep apnea syndrome}

\section{R Jokic, A Klimaszewski, M Crossley, G Sridhar, MF Fitzpatrick}

Objectives. The aim of this study was to compare the relative efficacy of continuous positive airway $\frac{5}{0}$ pressure (CPAP) and positional treatment in the management of positional obstructive sleep apnoea $\overrightarrow{i n}$ (OSA), using objective outcome measures. Design. A prospective, randomized, single blind crossover if comparison of CPAP and positional treatment for 2 weeks each. Setting. A university teaching hospital.

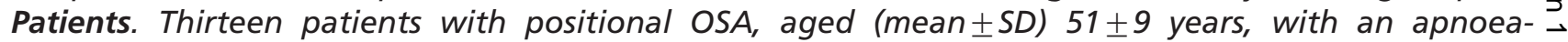

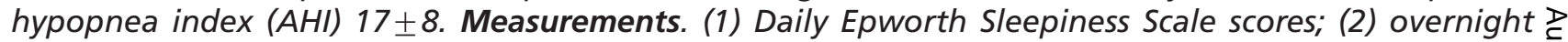
polysomnography, an objective assessment of sleep quality and $\mathrm{AHI}$; (3) maintenance of wakefulness testing; (4) psychometric test battery; (5) mood scales; (6) quality of life questionnaires; and (7) individual $\stackrel{\infty}{N}$ patient's treatment preference. Results. Positional treatment was highly effective in reducing time spent $\stackrel{0}{\circ}$ supine (median, 0; range, 0 to $32 \mathrm{~min}$ ). The AHI was lower (mean difference, 6.1; 95\% confidence interval $[\mathrm{Cl}] 2$ to $10.2 ; \mathrm{p}=0.007)$, and the minimum oxygen saturation was higher $(4 \% ; 95 \% \mathrm{Cl}, 1 \%$ to $8 \% ; p=0.02$ ) on CPAP compared with positional treatment. There was no significant difference, however, in sleep architecture, Epworth Sleepiness Scale scores, maintenance of wakefulness testing, sleep latency, psychometric test performance, mood scales, or quality of life measures. Conclusion. Positional treatment $\mathbb{\otimes}$ and CPAP have similar efficacy in the treatment of patients with positional OSA. (Chest 1999;115: 771-81)

Nasal continuous positive airway pressure

"The effectiveness of continuous positive airway pressure in improving health outcomes has been poorly evaluated". So concluded a systematic review of the health effects of obstructive sleep annoea (OSA) in $1997^{1}$ which stimulated fierce debate邦 and raised concerns that the provision of treatment for OSA in the UK would be undermined. The review questioned the link between OSA and vascular disease and criticised previous randomised trials of the efficacy of continuous positive airway pressure (CPAP) therapy on the grounds that an oral placebo was an inadequate control. While it is accepted that the impact of OSA on vascular morbidity and mortality remains to be determined, unresolved questions about the effectiveness of CPAP in controlling daytime somnolence are now addressed by the randomised comparison of therapeutic versus sham (subtherapeutic) CPAP in obstructive sleep apnoea (OSA) by Jenkinson et al. ${ }^{4}$ Sham CPAP was provided using an identical device to the therapeutic system, set to deliver the lowest possible positive pressure (about $1 \mathrm{~cm} \mathrm{H}_{2} \mathrm{O}$ ) and incorporating a deliberate leak to minimise rebreathing. Although use of an oral placebo has been defended, ${ }^{2}$ the employment of a subtherapeutic control circumvents criticisms of previous studies, and a pilot study confirmed the safety of the sham approach. Contrary to a previous study of sham respiratory support using negative pressure ventila- tion, ${ }^{5}$ patients were able to use the subtherapeutic CPAP $\stackrel{\frac{0}{2}}{x}$ for most of the night but duration of use was lower than $\frac{\sigma}{3}$ in patients receiving therapeutic CPAP (4.6 versus 5.4 . hours). All recruits were naive to CPAP therapy and 3 were informed that the purpose of the study was to compare different levels of CPAP. OSA was diagnosed by the presence of 10 or more dips in $\mathrm{SaO}_{2}$ of $>4 \%$ per $\frac{\mathrm{D}}{\mathrm{O}}$ hour. The therapeutic CPAP level for each patient was determined using an autotitration device. Primary $\cong$ outcome measures were the Epworth sleepiness score $N$ (ESS), modified maintenance of wakefulness test $\underset{\omega}{\mathrm{\omega}}$ (MWT - in which response times are measured while $\underset{0}{\sigma}$ individuals are requested to resist sleep for 40 minutes 0 in a darkened room on four occasions throughout the $\bar{\varnothing}$ day), and two components of the SF-36 health status $\stackrel{?}{+}$ questionnaire (energy and vitality, and mental domain total score). The number of overnight hypoxaemic dips $\frac{\vec{D}}{\mathbb{D}}$ fell significantly in patients receiving therapeutic CPAP but was unchanged in those on sham CPAP, confirming $\cong$ the validity of the technique as control therapy. Both subjective (ESS) and objective measures of sleepiness 8 improved in those using therapeutic CPAP. There were gains in energy and vitality, general health perception 흘 and the mental component summary of the SF-36 questionnaire compared with the sham limb. Small improvements occurred in the subtherapeutic group, confirming a placebo effect, but these were dwarfed by increases in the therapeutic category. 
As usual there are several caveats when interpreting such data. Firstly, the patients recruited had around 30 hypoxaemic dips $/ \mathrm{h}$ - that is, moderate obstructive sleep apnoea. They were also middle aged and markedly sleepy (median ESS 16) before treatment was initiated. The results cannot therefore be extrapolated to younger less symptomatic patients with mild OSA, although a proportion with mild OSA do seem to benefit symptomatically from CPAP. ${ }^{6}$ Secondly, the study was short term and specifically looked at measures of sleepiness so that the long term impact on mortality and cardiovascular and cerebrovascular health remains unclear and cannot be used to justify prescription. Important evidence on morbidity is likely to emerge from the Sleep Heart Health Study funded by the US National Heart, Lung and Blood Institute in the next few years. Finally, for reasons which were not discussed, only men were recruited to the study. There is no reason to suspect that women with OSA respond differently to CPAP physiologically, although presenting symptoms of OSA may vary slightly in women. ${ }^{7}$

Taking into account the results of this trial, the oral placebo controlled studies and a recent Spanish randomised study ${ }^{10}$ which showed that the combination of moderate weight loss plus CPAP offers greater symptomatic benefit than weight loss alone, it is now difficult to resist the conclusion that CPAP has a proven role in controlling sleepiness and improving quality of life in subjects with moderate OSA. This is of great clinical and public health importance, not least because OSA is associated with an increased risk of road traffic accidents. The Wisconsin Sleep Cohort study showed that patients with an AHI of $>15$ were more than seven times likely to have had multiple accidents. ${ }^{11}$ It is necessary for individuals in the UK with sleepiness as a result of OSA to notify the Driver and Vehicle Licensing Agency (DVLA) of their condition and these patients should not drive until their sleepiness has been adequately controlled. Available evidence suggests that impairment in simulated driving performance is successfully reversed by CPAP therapy. 1 . 13

\section{LIMITATIONS OF CPAP}

There are still a number of dilemmas concerning effective use of CPAP. These concern its role in mild OSA, frequent side effects, and compliance issues. In one study of patients with mild OSA (AHI 5-15) an improvement in symptom scores, subjective sleepiness, and quality of life was seen with CPAP therapy, but objective measures of sleepiness were unchanged and nightly use (average 2.8 hours) was significantly lower than in patients with more severe OSA. ${ }^{9}$

While daytime hypersomnolence, quality of life, and functional performance all improve with CPAP therapy, measures of sleepiness and cognitive impairment rarely return to normal. Numerous factors may contribute to persistent cognitive dysfunction including sleep fragmentation and reversible and irreversible cerebral damage due to hypoxaemia. It is not clear whether this failure to correct functional disturbance and alertness is due to the fact that CPAP therapy is inadequate or that its application is suboptimal, as many patients use it for only part of the night. In another sham CPAP controlled study ${ }^{14}$ both CPAP and sham CPAP had similar effects on sleep architecture, which was contrary to the expectation that sleep pattern and efficiency would be better with therapeutic CPAP. This study lasted only seven days, however, and it is possible that sleep architecture improves over a longer period of time as a result of better acclimatisation to the mask and machine.

Compliance with CPAP has long been regarded as 컥 imperfect, although it is difficult to know what is the "optimal dose" of CPAP. In the study by Jenkinson et $\stackrel{x}{.}$ $a l^{4}$ two of the 54 patients (4\%) did not take up CPAP. $\overrightarrow{\vec{F}}$ Long term use was not examined but discontinuation $\overrightarrow{0}$ rates in other studies have been shown to be around $\frac{\bar{\sigma}}{\sigma}$ $30-40 \%$. McCardle et $a l^{15}$ found that $68 \%$ of patients $\overline{\bar{\sigma}}$ with OSA were still using CPAP at three years. This $\underset{\mathbb{Q}}{\not}$ percentage increased to $86 \%$ in those with an ESS of $>10$ and AHI of $>30$. Early comprehensive technical o support tends to improve compliance, and this is better $\vec{\circ}$ in those patents who initiate referral themselves rather $\overrightarrow{\vec{\omega}}$ than those who are persuaded to seek treatment by their $\stackrel{\vec{\sigma}}{\sigma}$ partners, ${ }^{16}$ which shows that there are limits to altruism. Frequently reported problems associated with CPAP $\frac{\text { Oे }}{2}$ include inconvenience, nasal stuffiness, mask dis- iv comfort, claustrophobia, noise, and social/psychological on difficulties.

For all these reasons, alternatives to CPAP therapy 음 have been actively sought. The mandibular advance-. ment splint and positional management usefully extend is treatment options. Interesting new information emerges or from the studies by Ryan et $a l^{17}$ and Jokic et $a l^{18}$ which sheds light on their mechanism of action and effect- $\overrightarrow{ }$ iveness relative to CPAP.

\section{Mandibular advancement splint (MAS)}

Oral devices, constructed with the aim of maintaining airway patency during sleep, have existed for almost a century. An early version for correcting retrognathia was described by Pierre Robin in $1934 .{ }^{19}$ The exploration of oral positioning devices gained pace in the early 1980s but was overshadowed by the rapid development of $\stackrel{\mathbb{Q}}{2}$ CPAP. There have now been several randomised cross- $\overrightarrow{\overrightarrow{0}}$ over studies ${ }^{20-23}$ comparing the mandibular advance- 3 ment splint with CPAP in patients with OSA (table 1). Nearly all devices fit over the upper and lower teeth with the goal of positioning the mandible more anteriorly by gradual adjustment. Cumulative evidence suggests that both the MAS and CPAP reduce the AHI in patients with mild to moderate OSA, but the decrease achieved with CPAP is usually superior. Sleepiness scores also fall, but here again CPAP may be more effective than the MAS. A proportion of patients prefer the MAS 0 because of its greater convenience, and in some ? studie ${ }^{2 / 22}$ this applied to most subjects. On the other hand, there were individuals who could not tolerate the MAS and preferred CPAP.

As the MAS lifts the mandible forward it has been $N$ assumed that the device works by increasing the cross $\underset{\omega}{N}$ sectional area of the oropharynx and is therefore most 0 likely to be successful in patients with airway obstruction 6 at the oropharyngeal level. Such subjects comprise the minority of those with OSA, as by far the most common ? level of obstruction is at the velopharynx (soft palate), although a substantial proportion of patients with OSA have multi-segment collapse, particularly if they are obese. The article by Ryan et $a l^{17}$ is important as it $\stackrel{\mathbb{Q}}{2}$ challenges the preconception that the MAS improves oropharyngeal dimensions. In the group of patients 8 studied, use of the MAS during wakefulness increased the cross sectional area of the velopharynx but not that of the oropharynx. Moreover, the reduction in AHI $?$ recorded during polysomnography was correlated with the increase in cross sectional area of the velopharynx. A greater increase was seen in the lateral than in the anteroposterior dimension, hence normalising the con- 
Table 1 Review of crossover studies of CPAP therapy versus the mandibular advancement splint and positional management in OSA

\begin{tabular}{|c|c|c|c|c|c|c|c|c|c|c|}
\hline \multirow[t]{2}{*}{ Reference } & \multirow{2}{*}{$\begin{array}{l}\text { No. of } \\
\text { patients }\end{array}$} & \multirow{2}{*}{$\begin{array}{l}\text { Study } \\
\text { design }\end{array}$} & \multirow{2}{*}{$\begin{array}{l}\text { Duration } \\
\text { each limb }\end{array}$} & \multicolumn{3}{|l|}{ Mean AHI } & \multicolumn{3}{|l|}{ ESS } & \multirow[t]{2}{*}{ Pt preference } \\
\hline & & & & Baseline & MAS & CPAP & Baseline & MAS & CPAP & \\
\hline Clark $^{20}$ & 21 & $\begin{array}{l}\text { MAS v } \\
\text { CPAP }\end{array}$ & 2 weeks & $33.9(14.3)$ & $19.9(12.7)$ & $11.1(3.9)$ & - & - & - & \multirow{3}{*}{$\begin{array}{l}17 / 21 \\
\text { continued } \\
\text { with MAS } \\
\text { Of treatment } \\
\text { successes, } 6 \\
\text { preferred } \\
\text { MAS, } 1 \text { CPAP } \\
12 \text { MAS, } 6 \\
\text { CPAP }\end{array}$} \\
\hline Ferguson ${ }^{21}$ & 27 & $\begin{array}{l}\text { MAS v } \\
\text { CPAP }\end{array}$ & 4 months & $\begin{array}{l}\text { MAS: } 19.7 \\
(13.8) \\
\text { CPAP: } 17.6 \\
(13.2)\end{array}$ & $9.7(7.3)$ & $3.6(1.7)$ & \multicolumn{3}{|c|}{$\begin{array}{l}\text { 'MAS not as effective as CPAP' } \\
(p<0.05)\end{array}$} & \\
\hline Ferguson ${ }^{22}$ & 24 & $\begin{array}{l}\text { MAS v } \\
\text { CPAP }\end{array}$ & 4 months & $26.8(11.9)$ & $13.6(14.5)$ & $4.2(2.2)$ & 10.3 & 4.7 & 5.1 & \\
\hline Jokic ${ }^{18}$ & 13 & $\begin{array}{l}\text { Positional } \\
\text { v CPAP }\end{array}$ & 2 weeks & $\begin{array}{l}\text { Baseline } \\
17(8)\end{array}$ & $\begin{array}{l}\text { Positional } \\
9.5 \text { (1.9) }\end{array}$ & $\begin{array}{l}\text { CPAP } \\
3.4(0.5)\end{array}$ & $\begin{array}{l}\text { Baseline } \\
13(1.3)\end{array}$ & $\begin{array}{l}\text { Positional } \\
10\end{array}$ & $\begin{array}{l}\text { CPAP } \\
9\end{array}$ & $\begin{array}{l}4 \text { positional, } \\
7 \text { CPAP, } 2 \text { no } \\
\text { preference }\end{array}$ \\
\hline
\end{tabular}

figuration of the pharynx which tends to be elliptical in normal subjects but more spherical in patients with OSA. These findings fit well with the clinical observation that the MAS tends to produce beneficial results in a wide range of patients with mild to moderate OSA, including those who do not have primary obstruction at the oropharyngeal level.

As the authors discuss, a major limiting factor is that the imaging with and without the MAS was carried out while the patients were awake. It is well known that loss of pharyngeal muscle activity during sleep is a key determinant of airway collapse, and therefore it is possible that imaging studies during sleep might give different results. However, preliminary work ${ }^{24}$ elsewhere has confirmed that the MAS increases the cross sectional area of the upper airway during sleep and decreases pharyngeal compliance. It is hypothesised that anterior displacement of the mandible by the MAS pulls the tongue forward, thereby tensing the palatopharyngeus and palatoglossal muscles. Their combined action increases the lateral diameter of the pharynx which decreases upper airway compliance, thereby reducing collapsibility during sleep. Ryan et al used a two piece adjustable appliance (Klearway ${ }^{\mathrm{TM}}$ ); and one of the difficulties in comparing data on oral devices is that a huge variety of designs is employed. However, since the overall aim is to advance the mandible, generalisation is possible and it seems that the greater the degree of advancement (achieved by gradual adjustment), the better the outcome. ${ }^{25}$

\section{Positional management of OSA}

It should be recognised that, despite offering symptomatic benefit, CPAP and, to a lesser extent, the MAS are hardly unobtrusive. Patients and their medical attendants have been keen to seek simpler long term solutions which do not involve a major change in lifestyle or dependence on cumbersome equipment. Clearly, weight loss, cessation of smoking, and sleep hygiene advice will always have a role where appropriate, but positional management during sleep is an attractive complementary proposition.

There is a sound underlying logic to the strategy of persuading a snorer or patient with OSA to sleep in the lateral body position because snoring and obstructive events are increased when lying supine. Even during wakefulness, supraglottic airway resistance is higher when supine than erect. During sleep this effect is compounded by a further fall in lung volume and re- duced upper airway muscle activity. Mechanisms which 음 affect the upper airway during sleep are poorly un- $\rightarrow$ derstood, but it seems plausible that gravity dependent is backward movement of the tongue is less likely to occur or

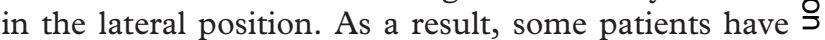
a clear positional variation in AHI in the supine and $\vec{\longrightarrow}$ lateral position. This differential effect is lost in those with severe OSA and in markedly obese patients. Positional OSA is characterised by a $50 \%$ reduction in $\vec{N}$ AHI from the supine to lateral position. Debate exists 8 regarding the prevalence of positional OSA (pOSA), which has been variously estimated to affect $9-60 \%$ of OSA patients. ${ }^{26}$ More recent studies tend to confirm a figure of $20-55 \%,{ }^{27}$ although referral bias may play a $\bar{O}$ part. Patients with pOSA have been shown consistently

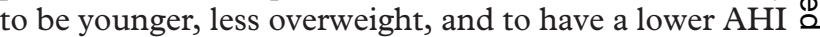
than their non-positional counterparts (factors which $\overrightarrow{\hat{0}}$ are inevitably interrelated). For effective results it is 3 obviously insufficient to reduce AHI by $50 \%$ in the lateral position if this $50 \%$ reduction only decreases the AHI from, say, 100 to 50 . A more rigorous clinical definition of pOSA is a $50 \%$ reduction in OSA from $\frac{0}{2}$ the supine to lateral position with AHI in the lateral position of $<15$ during a minimum duration of one hour 3 of sleep in the lateral position, including at least one period of rapid eye movement (REM) sleep.

Jokic et $a l^{18}$ have revived the idea of sewing a cotton $\bigcirc$ reel into the back of the pyjamas by using a backpack containing a soft ball (total size $10 \times 5.5$ inches) to $\frac{7}{0}$ discourage patients from sleeping on their back, and compared this intervention with CPAP in a two week o crossover study in 13 patients with mild pOSA. The $\tilde{O}$ authors hypothesised that some degree of persisting sleepiness and cognitive impairment in CPAP users 0 might be attributable to the CPAP machine itself causing 0 sleep disruption, and that positional modification would be less likely to disturb sleep. As well as measuring the? impact on $\mathrm{AHI}$ and nocturnal $\mathrm{Sao}_{2}$, this is the first report to detail the effects of positional therapy on functional outcomes such as daytime alertness and cognitive performance.

Importantly, the authors were able to show that the simple backpack device was very effective at maintaining 8 patients in the lateral sleep position (mean total sleep time 355 minutes, mean time supine 6 minutes). Both positional treatment and CPAP reduced AHI and im- ?्? proved minimum $\mathrm{SaO}_{2}$, but the effects were greater with CPAP (table 1). The two treatments were equally effective at decreasing the ESS and had a similar impact on the maintenance of wakefulness test (MWT), quality 
of life, and psychometric measures. However, contrary to the underlying hypothesis, there was no difference in sleep architecture or arousals during positional management or when using CPAP. An unusual feature of the study was that maintenance of wakeful time was within the normal reference range after positional and CPAP therapy, but unfortunately no baseline pretreatment measurement of MWT was carried out for comparison. Interestingly, at the start of the trial most patients expressed the hope that the positional management would be more effective, but by the end of the study seven preferred CPAP, four the positional device, and two had no preference. The authors conclude that simple positional management offers similar clinical efficacy to CPAP (at least short term) in patients with pOSA. It should be noted, however, that only a relatively small number of patients was studied, increasing the possibility of a type II statistical error - that is, inadequate power to exclude a small difference between treatments.

The study raises the question of whether positional management works long term, and also whether a training effect develops, as proposed by Cartwright, ${ }^{28}$ the pioneer of positional management of OSA. Using a similar backpack, Freebeck and Stewart ${ }^{29}$ showed that the time spent sleeping supine was reduced to less than $5 \%$ of total sleep time and $80 \%$ of patients achieved an $\mathrm{AHI}$ of less than 5 . In a subsequent study ${ }^{30}$ they reported that 17 of 18 patients complied with positional therapy and, even after reducing use of the backpack to one night per week, supine sleep time was less than $10 \%$ of total sleep time on nights without the pack which suggests that modification of sleep position without the positional device can be maintained, at least in the short term.

\section{Which treatment for what patient?}

These studies are helpful in deciding which option is most suitable for individual patients. Unequivocally, CPAP therapy is the treatment of choice for patients with moderate or severe OSA. In symptomatic patients with mild to moderate OSA, CPAP or the MAS can be considered. It is not possible to use the MAS in edentulous patients, those with severe periodontal disease, temporomandibular joint problems, or in children with growing dentition. Crossover studies have consistently shown that CPAP is slightly more effective than the MAS and therefore, for individuals with incomplete symptom relief with the MAS, CPAP should be explored. The MAS is also an option in patients with severe OSA who cannot tolerate CPAP, although long term outcome studies are lacking. In a recent prospective uncontrolled study of 21 patients with severe OSA (AHI 50-115), ${ }^{31}$ use of a MAS reduced the AHI to $<10$ in six patients, in 13 the AHI was reduced by more than $50 \%$, and only two patients showed no improvement. Compliance with the MAS is much more difficult to quantitate than CPAP use, and it is possible that long $\overrightarrow{\vec{*}}$ term discontinuation rates are high. Pancer $e t a l^{31}$ found that, after an average follow up of about one year, $86 \%$ c of patients reported continued nightly use, $60 \%$ were satisfied with the MAS, $13 \%$ were dissatisfied, and $10 \% \mathbb{D}$ were lost to follow up. The most common side effects were teeth discomfort in $32 \%$ and jaw discomfort in $26 \%$ of patients. Over long periods of time it is it is $\vec{\circ}$ possible that the MAS may cause occlusal changes or $\overrightarrow{\vec{\omega}}$ even mandibular remodelling, ${ }^{25}$ and therefore it is crit- $\stackrel{\omega}{\sigma}$ ical that adequate follow up is carried out with attention $\overrightarrow{\overrightarrow{5}}$ to these issues as well as control of sleep disordered breathing.

Positional management clearly requires further assess- of ment and is only curative in those with true positional $\stackrel{\infty}{5}$ OSA. To identify such individuals it is necessary for 0 multichannel diagnostic screening methods to in- $\overrightarrow{.}$ corporate a reliable body position monitor - this is not is possible using simple oximetry alone to establish the of

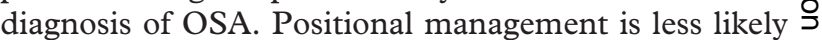
to be successful in obese patients who may not sense $\vec{\longrightarrow}$ the backpack, or if used with a soft mattress. It is conceivable that different positional devices have different success rates, and individual variation in tolerance $\stackrel{\vec{N}}{\mathbf{N}}$ is also probable. Oksenberg and Silverberg ${ }^{26}$ have de- 8 scribed an individual who managed to sleep on his back . with two tennis balls and a baseball in place! pOSA should be seen as a dynamic situation in which the use of alcohol, sedation, or weight gain may convert a positional OSA patient to a non-positional type.

To complicate matters, although the above treatments $\stackrel{\mathbb{Q}}{\varrho}$ serve many patients well, there is an increasing trend $\overrightarrow{\vec{D}}$ to combine modes - for example, positional man- 3 agement with the MAS or positional management with CPAP - allowing use of a lower therapeutic level of CPAP. ${ }^{32}$ Some patients prefer to use CPAP for most of the week and the MAS for short trips away from home. The combination of MAS and CPAP has been used in some units in patients who are unable to tolerate high 3 CPAP levels. The cost of dental construction of a mandibular advancement splint is broadly similar to a CPAP machine, but positional management is obviously cheaper. At present we have little knowledge about the "equivalence" of the techniques - for example, is eight $\frac{7}{2}$ hours of sleep using the MAS preferable to four hours of CPAP, despite the fact that the MAS is less effective of than CPAP in controlling AHI? Studies looking at the $\mathbb{0}$ effects of combination approaches on AHI and sub- స్ట

\section{LEARNING POINTS}

* CPAP therapy reduces sleepiness and improves health status in patients with moderate OSA.

* The mandibular advancement oral device increases the cross sectional area of the upper 8 airway and the increase in size of the velopharynx is correlated with the reduction in the apnoea/hypopnoea index during sleep.

* The mandibular advancement splint gives similar results to CPAP in patients with mild OSA

* Positional management may be useful in patients with positional OSA. 
jective and objective sleepiness will be relatively easy to perform, but longer term evaluation of the impact on cardiovascular and cerebrovascular end points will be extremely difficult, especially as treatment permutations may change over time, overall risk varies between individuals, and we cannot be sure that simple indices such as AHI, arousals, or autonomic variables are the best indicators of vascular stress.

Over the last few years much has been learned about the effectiveness of CPAP, which is now confirmed as evidence-based medicine for the management of sleepiness in patients with moderate OSA. Similar questions are now being addressed with alternative treatments which hold out hope for patients with mild sleep disordered breathing and those who fail with CPAP. Clearly, any treatment is ineffective and wasteful of resources if it is not used, and a pragmatic approach demonstrating that $\mathrm{AHI}$ and symptoms are controlled and patient preference is taken into account seems reasonable, pending the results of further long term studies.

1 Wright J, Johns R, Melville A, et al. Health effects of obstructive sleep apnoea and the effectiveness of continuous positive airways pressure: a systematic review of the research evidence. BMF 1997;314:851-60.

2 Douglas NJ. Systemic review of the efficacy of nasal CPAP. Thorax 1998;53:414-5.

3 Stradling J. Sleep apnoea and the misuse of evidence-based medicine Lancet 1997;349:201-2.

4 Jenkinson C, Davies R J O, Mullins R, et al. Comparison of therapeutic and subtherapeutic nasal continuous positive airway pressure for obstructive sleep apnoea: a randomised prospective parallel trial. Lancet 1999:353:2100-5.

5 Shapiro SH, Ernst P, Gray-Donald K, et al. Effect of negative pressure ventilation in severe chronic obstructive pulmonary disease. Lance

6 Engleman HM, Martin SE, Deary IJ, et al. Effect of CPAP therapy on daytome finction in patients with mild sleep apnoea syndrome. Thorax 1997;52:114-9.

7 Ambrogetti A, Olson LG, Saunders NA. Differences in the symptoms of men and women with obstructive sleep apnoea. Aust NZ F Med $1991 ; 21: 863-6$

8 Engleman HM, Martin SE, Deary IJ, et al. Effect of continuous positive airway pressure treatment on daytime function in sleep apnoea/hypopnoea syndrome. Lancet 1994;343:572-5.

9 Engleman HM, Kingshott RN, Wraith PK, et al. Randomized placebocontrolled trial of continuous posotive airway pressure for mild sleep apnea/hypopnea syndrome. Am f Respir Crit Care Med 1999;159: apnea/hyp $461-7$.

10 Ballester E, Badia JR, Hernandez L, et al. Evidence of the effectiveness of continuous positive airway pressure in the treatment of sleep apnea/ hypopnea syndrome. Am f Respir Crit Care Med 1999;159:495-501.

11 Young T, Blustein J, Finn L. Sleep disordered breathing and motor vehicle accidents in a population-based sample of employed adults. Sleep 1997;20:608-13.

12 George CFP, Boudreau AC, Smiley A. Effect of nasal CPAP on simulated driving performance in patients with obstructive sleep apnoea. Thorax 1997;52:648-53.

13 Hack M, Davies RJO, Mullins R, et al. Randomised propective parallel trial of therapeutic versus subtherapeutic nasal continuous positive airway pressure on simulated steering performance in patients with obstructive sleep apnoea. Thorax 2000;55:224-31.

14 Loredo JS, Ancoli-Israel S, Dimsdale JE. Effect of continuous positive airway pressure versus placebo continuous positive airway pressure on sleep quality in obstructive sleep apnea. Chest 1999;116:1545-9.

15 McCardle N, Devereux GM, Heidarnejad H, et al. Long-term use of CPAP therapy for sleep apnea/hypopnea syndrome. Am 7 Respir Crit Care Med 1999;159:1108-14.

16 Hoy CJ, Vennelle M, Kingshott RN, et al. Can intensive support improve continuous positive airway pressure use in patients with sleep apnea/ hypopnea syndrome? Am f Respir Crit Care Med 1999;159:1096-110.

17 Ryan CF, Love LL, Peat D, et al. Mandibular advancement oral appliance therapy for obstructive sleep apnoea: effect on awake calibre of the velopharynx. Thorax 1999;54:972-7.

18 Jokic R, Klimaszewski A, Crossley $M$, et al. Positional treatment vs continuous positive airway pressure in patients with positional obstructive sleep apnea syndrome.

19 Robin P. Glossoptosis due to atresia and hypotrophy of the mandible. Am $\mathcal{F}$ Dis Child 1934;48:541-7.

20 Clark GT, Blumenfield I, Yoffe Nea. A crossover study comparin the efficacy of continuous positive airway pressure with anterior mandibular positioning devices on patients with obstructive sleep apnea. Chest 1996;109:1477-83.

21 Ferguson KA, Ono T, Lowe AA, et al. A randomized study of an oral appliance vs nasal continuous positive airway pressure in the treatment of mild-moderate obstructive sleep apnea. Chest 1996;109:1269-75.

22 Ferguson KA, Ono T, Lowe AA, et al. A short-term controlled trial of an adjustable oral appliance for the treatment of mild to moderate (1997:52:362-8.

23 Tan YK, L'Estrange PR, Grant HR, et al. A randomised crossover study of continuous positive airway pressure (CPAP) versus mandibular advancement splint (MAS) in mild and moderate obstructive sleep apnoea (OSA). Eur Respir f 1998;12(Suppl 28):5s

24 Oshima T, Vazquez JC, Masuda A, et al. Effects of incremental mandibular protrusion on cross-sectional area of the passive pharynx during medicated sleep in patients with obstructive sleep apnea. Am f Respir Crit Care Med 1997;155:A676.

25 Clark GT. Mandibular advancement devices and sleep disordered breathing. Sleep Med Rev 1998;2:163-74.

26 Oksenberg A, Silverberg DS. The effect of body posture on sleeprelated breathing disorders: facts and therapeutic implications. Sleep Med Rev 1998;2:139-62.

27 Oksenberg A, Silverberg DS, Arons E, et al. Positional vs nonpositional obstructive sleep apnea patients. Anthropomorphic, nocturnal polysomnographic and multiple sleep latency test data. Chest 1997;112: 629-39.

28 Cartwright C. New versions of old treatments: hope for the CPAP holdout. Sleep Med Rev 1998;2:135-7.

29 Freebeck P, Stewart D. Positional training while you sleep. Sleep Res 1995;24:235.

30 Freebeck P, Stewart D. Compliance and effective therapy for positional apnea. Sleep Res 1995;24:236.

31 Pancer J, Al-Faifi S, Al-Faifi M, et al. Evaluation of a variable mandibular advancement appliance for treatment of snoring and sleep apnea. Chest 1999;116:1511-8

32 Oksenberg A, Silverberg DS, Arons E, et al. The sleep supine position has a major effect on optimal nasal continuous positive airway pressure. Chest 1999;116:1000-6. 